\title{
Prism adaptation improves voluntary but not automatic orienting in neglect
}

\author{
Tanja C.W. Nijboer ${ }^{\mathrm{a}}$, Rob D. McIntosh ${ }^{\mathrm{b}}$, Gudrun M.S. Nys ${ }^{\mathrm{c}}$, H. Chris Dijkerman ${ }^{\mathrm{a}}$ and A. David Milner ${ }^{\mathrm{d}}$ \\ a'Department of Experimental Psychology, Utrecht University, Utrecht, The Netherlands, 'bchool of Philosophy, Psychology, and Language Sciences, \\ University of Edinburgh, Edinburgh, United Kingdom, 'Laboratory for Neuropsychology, Department of Internal Medicine, Neurology Section, \\ Ghent University, Belgium and ${ }^{\mathrm{d} D e p a r t m e n t}$ of Psychology, Durham University, Durham, United Kingdom \\ Correspondence to Tanja C.W. Nijboer, Utrecht University, Department of Experimental Psychology, Helmholtz Institute, Heidelberglaan 2, 3584 CS \\ Utrecht, the Netherlands \\ Tel: + 31302533572; fax: + 313025345II; e-mail: t.c.w.nijboer@uu.nl
}

Received 19 November 2007; accepted 24 November 2007

\begin{abstract}
Prism adaptation has been shown to temporarily ameliorate the symptoms of unilateral neglect. The underlying mechanisms of change are not yet fully understood. In this study, we investigate the influence of this treatment on attentional orienting under conditions of exogenous (peripheral onset) and endogenous (central symbolic) cueing. In one patient with left visual extinction and recovered neglect, and another patient with left visual neglect, visuo-motor adaptation to a rightward prismatic shift of $10^{\circ}$
\end{abstract}

Keywords: neglect, orienting of attention, prism adaptation improved leftward orienting of attention following an endogenous but not an exogenous cue; leftward re-orienting of attention was also improved in the endogenous task for the second patient. We suggest that prism adaptation may ameliorate neglect by improving compensatory processes of leftward voluntary orienting, rather than by a fundamental change in attentional bias. NeuroReport 19:293-298 (c) 2008 Wolters Kluwer Health । Lippincott Williams \& Wilkins.

\section{Introduction}

There is ample evidence that prism adaptation (PA) improves the clinical signs of neglect and performance on standard neglect tests (e.g. line bisection, line cancellation) [1-4]. Notwithstanding this evidence of beneficial effects, the underlying mechanisms of change are not well understood. PA has been found to rebalance the distribution of spatial attention by reducing the rightward bias, often considered to be the core deficit in neglect. This, however, has been observed when using a visual temporal order judgement task [5], but not when using a speeded visual search task [6]. In a study on eye movement behavior before and after PA [7], it was found that, although ocular exploration became more symmetrical after PA, the first saccade, arguably a measure of automatic orienting, remained directed rightward in the great majority of trials. In this study we aimed to determine whether PA improves automatic orienting, voluntary orienting, or both.

An important distinction in the attention literature is the one between exogenous and endogenous orienting [8]. Both forms of orienting have been studied extensively using variants of the Posner reaction time (PRT) task. In the original task $[9,10]$, participants were presented with three horizontally arranged boxes while fixating the central box, and responded by pressing a key when a target appeared in one of the other two boxes. Either a (predictive) central symbolic or a (nonpredictive) peripheral onset cue, indicating one of the two locations, preceded the target. Valid cues correctly predicted the box in which the target would appear, whereas invalid cues indicated the other box. In these tasks, participants show a RT advantage on valid trials compared with invalid trials. This suggests that the cue prompts attentional orienting toward the cued location, speeding up the processing of targets in that region, and slowing responses to targets at other locations [11].

Posner et al. [10] tested six right parietal brain-damaged (RBD) and seven left parietal brain-damaged (LBD) patients. These authors found that both groups had abnormally long RTs when a contralesional target was preceded by an invalid cue, but particularly the RBD patients [10,12,13]. These abnormally long RTs to invalidly cued contralesional targets did not occur in frontal, midbrain, or temporal control patients. A similar pattern has also been observed in neglect [14-16], and is sometimes attributed to specific problem disengaging attention from ipsilesional locations ('disengage deficit' [10]).

In this study, we investigated the influence of PA on attentional orienting using the PRT task, with either peripheral exogenous or central endogenous cues. Based on previous findings, we hypothesized that PA would improve leftward endogenous, but not exogenous, orienting. The experiments were conducted in accordance with the Declaration of Helsinki, with the understanding and written consent of all participants, and were approved by the local ethical committee.

\section{Methods}

Participants

Patient 1 was a 76-year-old right-handed male, who had suffered a hemorrhagic stroke in the right temporo-parietooccipital region (see Fig. 1a) 22 months before the present 
testing. He showed no motor deficits, or visual field deficits as tested by dynamic perimetry. He had exhibited clinical signs of neglect early after his stroke, but these had largely resolved according to standard tests [Line Bisection: 6/9; Star Cancellation: 51/54; Line Cancellation: 33/36; Representational Drawing: 3/3; Scene Copying: 4/5 (leftmost missed; 2 with left-sided details missing)]. On the Balloons test version B, 16 targets were cancelled, 6 of them on the left. Thus, mild neglect was still detectable, most clearly in complex scene and timed serial search tests. In addition, he still showed reliable visual and tactile extinction.

Patient 2 was a 66-year-old right-handed female, who suffered an acute subarachnoid hemorrhage 70 months before the present testing. She developed severe vasospasms, resulting in a large ischemic infarction of the right hemisphere (see Fig. 1b). Early neuropsychological testing had revealed perceptual impairment (visual perception and construction) and severe left-sided visual neglect. At the time of testing, she still showed neglect on standard tasks: Line Bisection: 3/9; Star Cancellation: 47/54; Letter Cancel- lation: 35/40; Line Cancellation: 30/36; Representational Drawing: 2/3; Figure and Shape Copying: 1/4. On visual confrontation, she missed all left-sided stimuli on both single and simultaneous stimulation. Perimetry at the time of testing indicated some improvement, but some stimuli were still missed in the left visual field. A left visual field defect could not be excluded or confirmed definitively on perimetric grounds, given the presence of visual neglect.

Eight right-handed healthy age-matched control participants were also tested (three females, five males; mean age: 67.9 years, SD: 5.5$)$.

\section{Procedure}

Both patients took part in three testing sessions: two before PA to establish baseline performance, and one immediately after PA. The sequence for each session was as follows: adaptation procedure, exogenous PRT task, endogenous PRT task, adaptation procedure, endogenous PRT task, exogenous PRT task. In the first two sessions, adaptation
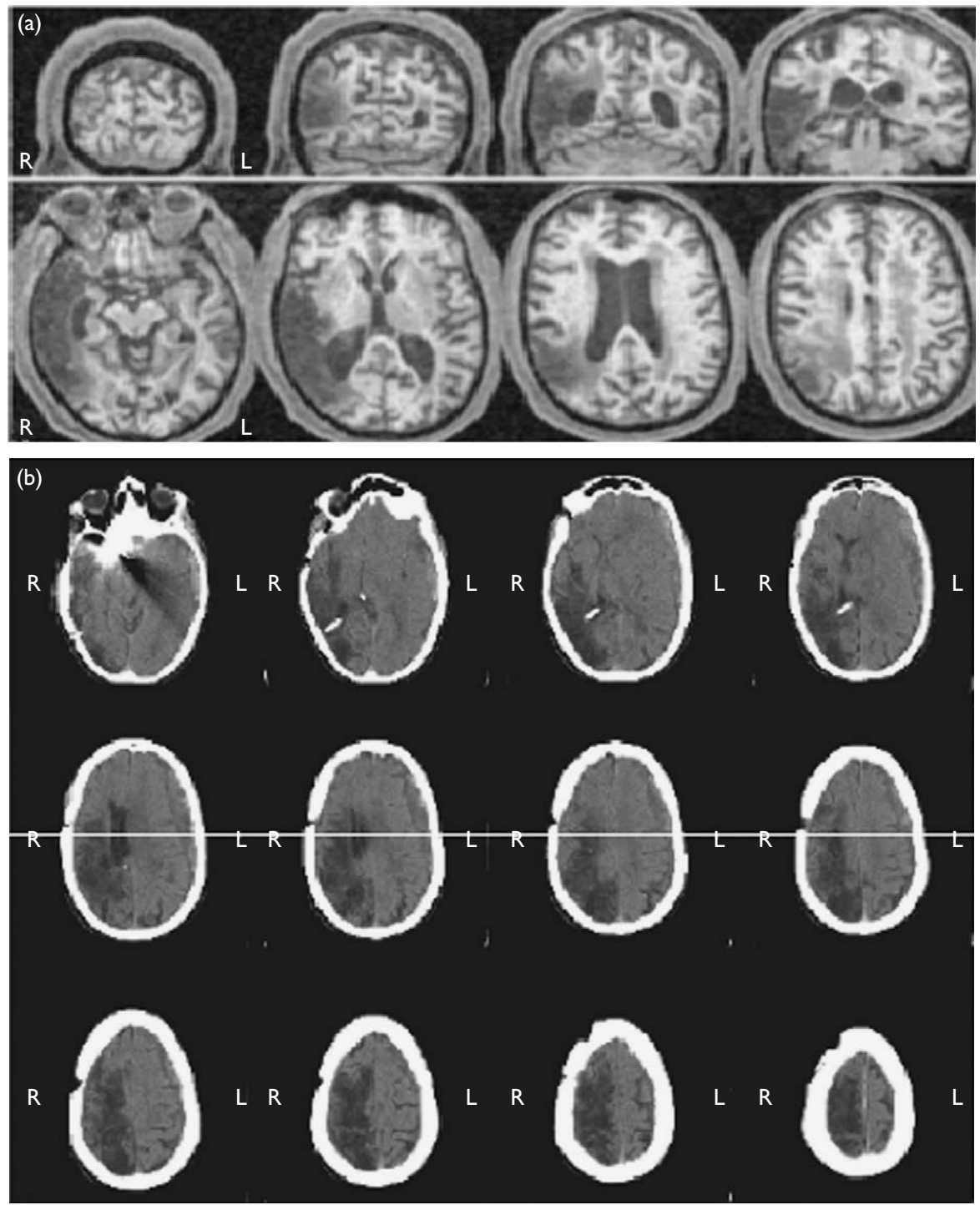

Fig. I Scans of patient I [(a) fMRI] and patient 2 [(b) CTscan]. fMRI, frontal magnetic resonance imaging; CT, computed topography. 
was a sham, using plain glasses; in the third session, $10^{\circ}$ rightward prisms were used (see below). Each testing session was performed on a separate day, and sessions were spaced approximately 4 days apart. The age-matched controls performed the PRT task once, without the previous adaptation procedure.

\section{PRT task}

Participants were seated in front of a 17 -inch $(43 \mathrm{~cm})$ computer monitor. The distance from eyes to the monitor was approximately $50 \mathrm{~cm}$. All stimuli were presented in white against a black background.

The participant initiated each trial by pressing the space bar. At the start of each trial, four dots $\left(0.68^{\circ}\right.$ of visual angle $)$ would converge, over $400 \mathrm{~ms}$, from the corners of a virtual square $\left(3.43^{\circ}\right)$ at the center of the screen, to draw attention to this location. At the point of convergence, the dot was replaced by a fixation cross $\left(0.68^{\circ}\right)$. After $1000 \mathrm{~ms}$, the fixation cross disappeared, and a cue was presented. In the exogenous condition, this consisted of four corners of a $2.75^{\circ}$ square (corners: $0.92^{\circ}$ horizontally and vertically), centered $6.28^{\circ}$ left or right of the screen center. In the endogenous condition, two arrowheads ('or', $1.72^{\circ}$ total length) were presented centrally. The target appeared following a cuetarget onset asynchrony (CTOA) that varied randomly between 100 and $200 \mathrm{~ms}$ in the exogenous condition and between 300 and $700 \mathrm{~ms}$ in the endogenous condition. The target was a dot $\left(0.29^{\circ}\right), 6.28^{\circ}$ to the left or right of the screen center. Participants responded by pressing the spacebar with the right hand as soon as they saw the target. Both target and cue remained visible until the participant responded, or for $3000 \mathrm{~ms}$ at most. To control for guessing, $10 \%$ of trials in each condition were catch trials, in which the cue was not followed by a target. If the participant responded on a catch trial, they received an error message in the center of the screen.

In the exogenous condition, the target appeared at the cued location $50 \%$ of the time; the cues were thus noninformative regarding target location. In the endogenous attention, the cues were predictive, and validly predicted the target location on $70 \%$ of noncatch trials. The composition of a trial block was as follows:

(a) exogenous condition: 220 trials: 50 target left, valid cue; 50 target right, valid cue; 50 target left, invalid cue; 50 target right, invalid cue; 20 catch trials (10 cue left, 10 cue right);

(b) endogenous condition: 374 trials: 120 target left, valid cue; 120 target right, valid cue; 50 target left, invalid cue; 50 target right, invalid cue; 34 catch trials (17 cue left, 17 cue right).

The four blocks in each session formed an ABBA design, for a total of 1188 experimental trials. Fifty practice trials were given before the first block of each condition, and ten before the second block in each condition.

\section{Prism adaptation procedure}

The PA procedure was based on that used by Rossetti et al. [1]. Patients wore a pair of goggles fitted with wide-field point-to-point prismatic lenses, inducing a rightward optical shift of $10^{\circ}$. Exposure consisted of 100 fast pointing movements made to visual targets presented $10^{\circ}$ to the left or right of the body midline at a distance of $65 \mathrm{~cm}$, with 50 responses made to each in a random order. A board was held under the patient's chin to prevent viewing of the hand at its starting position, but allowing an unobstructed view of the pointing targets and terminal errors. Following prism exposure, an open-loop pointing task was used to check informally for a prismatic after-effect. Both patients showed the expected after-effect: a leftward shift of about $3 \mathrm{~cm}$. The sham adaptation procedure was identical except that plain glasses were used.

\section{Data analysis}

Catch trials, misses, and trials with RTs less than $150 \mathrm{~ms}$ were removed. The rest of the data were trimmed by blockwise removal of outliers ( $>2$ SDs from block mean).

The mean RTs of the control group were analyzed using separate repeated-measures ANOVAs for the exogenous and endogenous conditions, with target side (left, right) and cue validity (valid, invalid) as factors. For the patients, separate between-subjects ANOVAs were performed for the exogenous and endogenous conditions, with target side (left, right), cue validity (valid, invalid) and session (1, 2, and 3) as factors.

\section{Results}

Controls

The controls showed shorter RTs to validly cued targets, for both the exogenous and endogenous conditions $[\mathrm{F}(1,7)=8.8$, $P<0.02 ; \mathrm{F}(1,7)=12.0, P<0.01$, respectively]. In the exogenous condition, there was also an effect of target side $[F(1,7)=17.9, P<0.01]$, reflecting shorter RTs to right-sided targets. This may be a stimulus-response compatibility effect: since the manual response was made with the right hand, responses tended to be faster for stimuli appearing in the right visual field. The same trend was seen in the endogenous condition, but was not reliable. There was no reliable interaction between target side and cue validity.

\section{Patients}

\section{Exogenous attention}

The left panels of Fig. 2 illustrate the performance of the patients in the exogenous condition; the accompanying statistical analyses are summarized in the left column of Table 1. As expected, both patients had reliably longer RTs to left-sided targets, and to invalidly cued targets. Only Patient 2 exhibited a reliable interaction of side by validity, such that RTs were differentially longer for invalidly cued targets on the left (i.e. disengagement deficit). Patient 2 additionally showed a reliable effect of session, and a reliable interaction of session by side: this reflected longer RTs in session 1 than in sessions 2 or 3 , particularly on the left. Importantly, neither patient showed any effects involving session that could be attributed to prism adaptation.

\section{Endogenous attention}

The right panels of Fig. 2 illustrate the performance of the patients in the endogenous condition; the accompanying statistical analyses are summarized in the right column of Table 1. Again, both patients had reliably longer RTs to leftsided targets, and to invalidly cued targets. In this task, both patients showed a pronounced disengagement deficit, as indexed by the interaction of side by validity. Both patients showed a reliable effect of session, with reduced RTs in the 

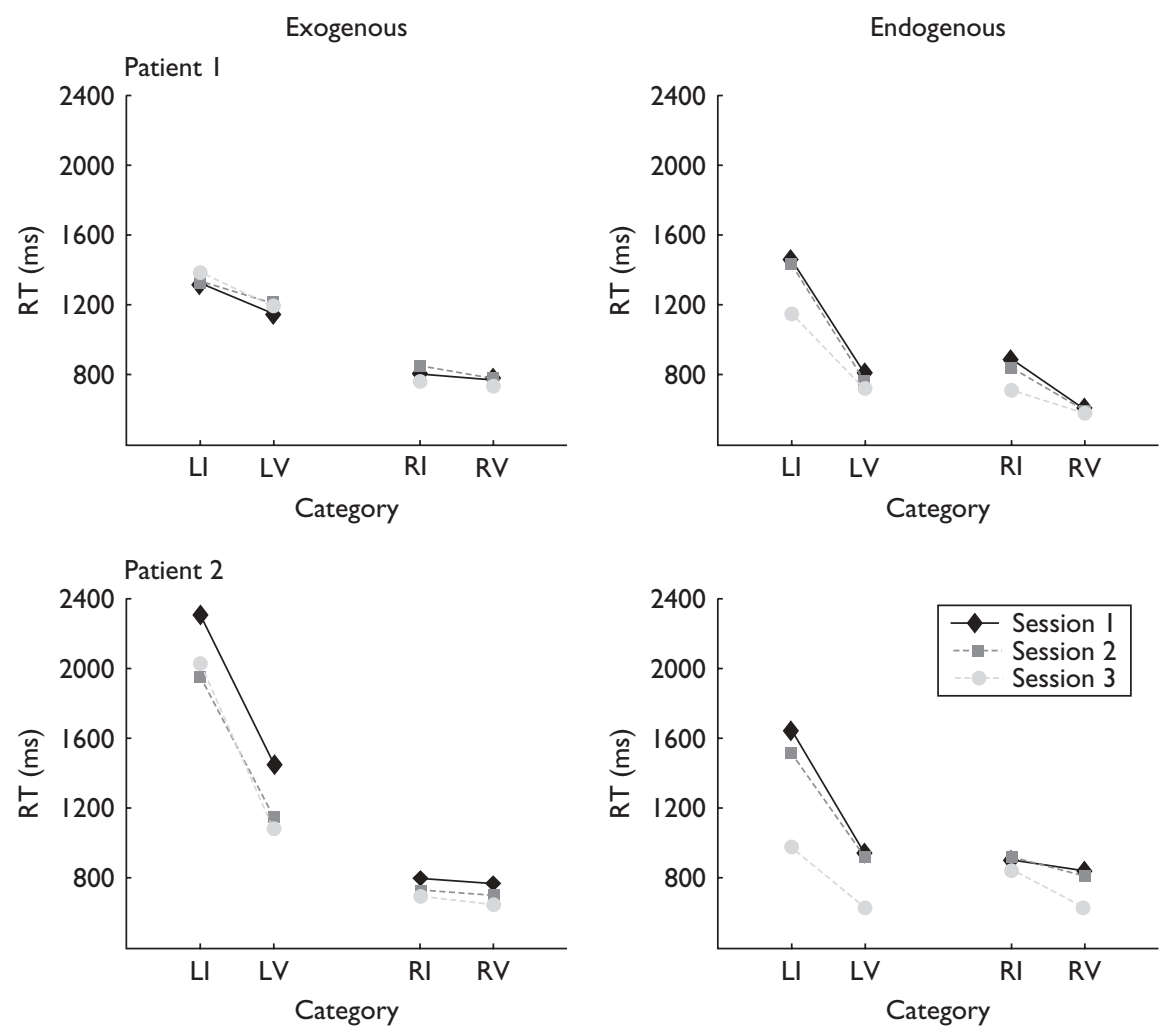

Fig. 2 Mean RTs of patient I (top row) and patient 2 (bottom row). The left panels show the exogenous condition and the right panels the endogenous condition. Trial category is coded by target side and cue validity (LI, left invalid; LV, left valid; RI, right invalid; RV, right valid). Sessions I and 2 are preprism adaptation; session 3 is post-prism.

Table I ANOVA results for both patients in the exogenous and endogenous conditions

\begin{tabular}{|c|c|c|}
\hline & Exogenous & Endogenous \\
\hline \multicolumn{3}{|l|}{ Patient I } \\
\hline Session & $\mathrm{F}(2, I 174)=0.91, P=0.4 \mathrm{I}$ & $\begin{array}{c}F(2,1999)=27.49 \\
P<0.001\end{array}$ \\
\hline Side & $\begin{array}{c}F(I, I 174)=670.13 \\
P<0.001\end{array}$ & $\begin{array}{c}F(I, 1999)=415.76 \\
P<0.001\end{array}$ \\
\hline Validity & $\begin{array}{c}F(I, I 174)=6.44 \\
P<0.05\end{array}$ & $\begin{array}{c}F(I, 1999)=542.13 \\
P<0.001 \\
F(2.1999)=3.30\end{array}$ \\
\hline Session $\times$ side & $\mathrm{F}(2, I 174)=2.18, P=0.11$ & $\begin{array}{c}P<0.05 \\
F(2,1999)=12.29\end{array}$ \\
\hline Session $\times$ validity & $F(2, I \mid 74)=0.07, P=0.94$ & $\begin{array}{c}P<0.00 I \\
F(I, 1999)=I 15.09\end{array}$ \\
\hline $\begin{array}{l}\text { Side } \times \text { validity } \\
\text { Session } \times\end{array}$ & $\mathrm{F}(\mathrm{I}, \mathrm{II} 74)=0.15, P=0.70$ & $P<0.001$ \\
\hline side $\times$ validity & $F(2, I I 74)=0.74, P=0.48$ & $\mathrm{~F}(2,1999)=1.10, P=0.33$ \\
\hline \multicolumn{3}{|l|}{ Patient 2} \\
\hline Session & $\begin{array}{c}\mathbf{F}(2, I 148)=23.43 \\
P<0.001\end{array}$ & $\begin{array}{c}\mathbf{F}(2,1866)=119.97 \\
P<0.001\end{array}$ \\
\hline Side & $\begin{array}{c}F(I, I \mid 48)=1236.3 I \\
P<0.00 I \\
F(I, I \mid 48)=282.8 I\end{array}$ & $\begin{array}{c}F(I, I 866)=248.96 \\
P<0.00 I \\
F(I, I 866)=366.85\end{array}$ \\
\hline $\begin{array}{l}\text { Validity } \\
\text { Session } \times \text { side }\end{array}$ & $\begin{array}{c}P<0.001 \\
F(2, I 148)=7.92 \\
P<0.001\end{array}$ & $\begin{array}{c}P<\mathbf{0 . 0 0 1} \\
\mathbf{F}(2,1866)=37.48 \\
P<\mathbf{0 . 0 0 1} \\
F(2,1866)=2.83\end{array}$ \\
\hline Session $\times$ validity & $\begin{array}{c}F(2, I \mid 48)=0.91, P=0.40 \\
F(2, I 148)=243.1\end{array}$ & $\begin{array}{c}P=0.06 \\
F(I, \mid \mathbf{8 6 6})=\mathbf{1 4 0 . 7 0}\end{array}$ \\
\hline Side $\times$ validity & $P<0.001$ & $P<0.001$ \\
\hline $\begin{array}{l}\text { Session } \times \\
\text { side } \times \text { validity }\end{array}$ & $\begin{array}{c}F(2, I 148)=0.38 \\
P=0.68\end{array}$ & $\begin{array}{c}F(2,1866)=17.70, \\
P<0.001\end{array}$ \\
\hline
\end{tabular}

post-prism session (session 3). For both patients, the interaction of session by side was highly reliable, reflecting a differential improvement of endogenous orienting to the left following prism adaptation. Patient 2 additionally showed a reliable three-way interaction of session by side by validity, indicating the disengagement deficit was also improved following prism adaptation in this patient.

\section{Discussion}

Age-matched controls showed the expected effects of cue validity on both sides in both conditions, thus confirming the suitability of our experimental tasks. The two patients showed slowed responses to left-sided targets before PA. In addition, a differential slowing of responses to invalidly cued contralesional targets (disengagement deficit) was observed for patient 1 in the exogenous condition, and for both patients in the endogenous condition. These patterns are consistent with previous results from unilaterally braindamaged patients $[9,10,14-16]$.

Our main finding was a clear improvement of contralesional orienting in the endogenous condition following PA in both patients, with no comparable effects in the exogenous condition. This is compatible with the suggestion that PA may particularly influence voluntary rather than automatic orienting of attention [7]. In other words, PA may enable patients to attend successfully to the left through conscious effort, despite their automatic orienting remaining biased rightward.

Comparisons of performance between our two conditions are complicated slightly by methodological differences 
between them. One obvious difference is that non-overlapping ranges of CTOAs were used: $100-200 \mathrm{~ms}$ for the exogenous, and 300-700 ms for the endogenous condition. These are typical ranges within which 'facilitatory' effects should arise for exogenous and endogenous cues respectively; as such, exogenous orienting is normally identified with fast orienting [9]. Nonetheless, it could be suggested that the different CTOA ranges, rather than the nature of the cues themselves, might critically underlie the differential effects of PA in our patients. One way to test this possibility would be directly to compare the effects of PA on endogenous and exogenous orienting within the longer CTOA range.

A second difference between conditions is in the location of the cues, with the exogenous cues being peripheral, and the endogenous cues central. Again, these task differences are standard within the literature. Several recent studies, however, have suggested that exogenous orienting need not arise solely through the 'pulling' of attention to a cued location. Attentional orienting can be induced by nonpredictive central arrow cues [17-19], suggesting that symbolic cues (provided they are highly familiar, such as arrow direction) may also direct attention in a rather automatic way. Therefore, we cannot exclude the possibility that our endogenous condition may have incorporated some automatic component. Accordingly, our finding that PA did not improve exogenous orienting must be taken to apply strictly to exogenous orienting to peripheral onsets. Clearly, it would be interesting to investigate whether PA enhances contralesional orienting to nonpredictive symbolic cues in patients with neglect.

Since contralesional orienting to peripheral events was refractory to PA in our patients, there may be some concern over the inability to exclude left visual field defects definitively in the clinical assessment of patient 2. Several considerations, however, suggest that field defects are unlikely to explain this patient's results. First, such an account would predict a general lack of influence of left exogenous cues, and thus a reduced effect of cue validity for right targets relative to healthy controls (and patient 1), which was not observed. Second, patient 2 rarely missed left-sided targets in either task, suggesting that they did not fall within a field defect. Finally, patient 2's performance across conditions was similar to that of patient 1 , for whom field defects were excluded.

Our observation that PA exerted its beneficial effects only within the timescale of voluntary orienting is consistent with the well-established effects of PA on clinical signs of neglect [1-4], as these are generally assessed through non-time-limited tasks (e.g. target cancellation, line bisection) in which voluntary orienting could compensate for a more primary bias of automatic attention. It also corresponds well with Dijkerman et al.'s observation that PA did not affect first saccade direction in a simple sizematching task, despite pronounced effects on later ocular exploration [7]. Two more recent studies, however, have reported an increase in leftward-directed initial saccades after PA [20,21]. Notably, however, both studies used reading tasks, in which the lexical nature of the stimuli may have constituted an overlearned cue for leftward orienting.

The present findings contrast with a report by Striemer and Danckert, who argued that PA did facilitate exogenous orienting in their RBD patients (with and without neglect), especially at a short CTOA (50 ms) [22]. Unfortunately, as only four patients were tested, in a repeated-measures design, the analysis did not have sufficient power to establish statistically that the benefit was specific to contralesional orienting. Nonetheless, PA has also been reported to rebalance visual temporal-order judgements in neglect patients [5], perhaps supporting the view that automatic orienting may be improved. It is quite possible that the effects of PA may vary between patients, maybe depending upon the relative sparing of ventral and dorsal attentional networks, associated with automatic and voluntary orienting, respectively [23].

In summary, this study demonstrated impaired contralesional orienting, following both exogenous and endogenous cues in two patients with elements of the neglect syndrome. Both patients showed a pronounced beneficial effect of PA in the endogenous condition, including a reduction of the disengagement deficit in one patient. By contrast, neither patient showed any effect of PA upon exogenous orienting. Although exogenous orienting to peripheral events is not necessarily refractory to PA in all patients [5,22], our data suggest that the beneficial effects of PA for the neglect syndrome may be mediated primarily via the facilitation of voluntary contralesional orienting.

\section{References}

1. Rossetti Y, Rode G, Pisella L, Farne A, Li L, Boisson D, Perenin M. Prism adaptation to a right optical deviation rehabilitates left hemispatial neglect. Nature 1998; 395:166-169.

2. Rode G, Rossetti Y, Boisson D. Prism adaptation improves representational neglect. Neuropsychologia 2001; 39:1250-1254.

3. Frassinetti F, Angeli V, Meneghello F, Avanzi S, Ladavas E. Long-lasting amelioration of visuospatial neglect by prism adaptation. Brain 2002; 125:608-623

4. McIntosh RD, Rossetti Y, Milner AD. Prism adaptation improves chronic visual and haptic neglect; a single case study. Cortex 2002; 38: 309-320.

5. Berberovic N, Pisella L, Morris AP, Mattingley JB. Prismatic adaptation reduces biased temporal order judgements in spatial neglect. NeuroReport 2004; 19:1199-1204.

6. Morris AP, Kritikos A, Berberovic N, Pisella L, Chambers CD, Mattingley JB. Prism adaptation and spatial attention: a study of visual search in normals and patients with unilateral neglect. Cortex 2004; 40:703-721.

7. Dijkerman HC, McIntosh RD, Milner AD, Rossetti Y, Tilikete C, Roberts RC. Ocular scanning and perceptual size distortion in hemispatial neglect: effects of prism adaptation and sequential stimulus presentation. Exp Brain Res 2003; 153:220-230.

8. Egeth HE, Yantis S. Visual attention: control, representation, and time course. Ann Rev Psychol 1997; 48:269-297.

9. Posner MI. Orienting of attention. Quar J Exp Psychol 1980; 32:3-25.

10. Posner MI, Walker JA, Friedrich FJ, Rafal RD. Effects of parietal injury on covert orienting of attention. J Neurosc 1984; 4:1863-1874.

11. Bartolomeo $\mathrm{P}, \mathrm{Chokron} \mathrm{S}$. Orienting of attention in left unilateral neglect. Neurosc Biobehav Rev 2002; 26:217-234.

12. Morrow LA, Ratcliff G. The disengagement of covert attention and the neglect syndrome. Psychobiology 1988; 16:261-269.

13. Losier BJW, Klein RM. A review of the evidence for a disengage deficit following parietal lobe damage. Neurosc Biobehav Rev 2001; 25: 1-13.

14. Farah MJ, Wong AB, Monheit MA, Morrow LA. Parietal lobe mechanisms of spatial attention: modality-specific or supramodal? Neuropsychologia 1998; 27:461-470.

15. Friedrich FJ, Egly R, Rafal RD, Beck D. Spatial attention deficits in humans: a comparison of superior parietal and temporal-parietal junction lesions. Neuropsychology 1998; 12:193-207.

16. Ladavas E, Carletti M, Gori G. Automatic and voluntary orienting of attention in patients with visual neglect: horizontal and vertical dimension. Neuropsychologia 1994; 32:1195-1208. 
17. Hommel B, Pratt J, Colzato L, Godijn R. Symbolic control of visual attention. Psycho Sc 2001; 12:360-365.

18. Tipples J. Eye gaze is not unique: automatic orienting in response to uninformative arrows. Psycho Bull Rev 2002; 9:314-318.

19. Ristic J, Kingstone A. Attention to arrows: pointing to a new direction. Quar J Exp Psychol 2006; 59:1921-1930.

20. Angeli V, Benassi MG, Ladavas E. Recovery of oculo-motor bias in neglect patients after prism adaptation. Neuropsychologia 2004; 42:1223-1234.
21. Serino A, Angeli V, Frassinetti F, Ladavas E. Mechanisms underlying neglect recovery after prism adaptation. Neuropsychologia 2006; 44: 1068-1078.

22. Striemer C, Danckert J. Prism adaptation reduces the disengage deficit in right brain damage patients. NeuroReport 2007; 18:99-103.

23. Corbetta M, Shulman GL. Control of goal-directed and stimulus-driven attention in the brain. Nat Rev Neurosc 2002; 3:201-215. 